\title{
The role of neutrophil lymphocyte ratio to lever- age the differential diagnosis of familial Mediter- ranean fever attack and acute appendicitis
}

\author{
Adem Kucuk ${ }^{1}$, Mehmet Fatih Erol², Soner Senel ${ }^{3}$ Emir Eroler ${ }^{2}$, Havvanur Alparslan Yumun ${ }^{2}$, \\ Ali Ugur Uslu ${ }^{4}$, Asiye Mukaddes Erol5, Deniz Tihan ${ }^{2}$, Ugur Duman $^{2}$, Tevfik Kucukkartallar ${ }^{6}$, \\ and Yalcin Solak, ${ }^{7, *}$
}

\begin{abstract}
${ }^{1}$ Division of Rheumatology, Department of Internal Medicine, Necmettin Erbakan University, Konya; ${ }^{2}$ Department of General Surgery, Bursa Sevket Yilmaz Research and Training Hospital, Bursa; ${ }^{3}$ Division of Rheumatology, Department of Internal Medicine, ${ }^{4}$ Department of Internal Medicine, Cumhuriyet University, Sivas; ${ }^{5}$ Department of Physical Medicine and Rehabilitation, Bursa Sevket Yilmaz Research and Training Hospital, Bursa; ${ }^{6}$ Department of General Surgery, Necmettin Erbakan University, Konya; ${ }^{7}$ Division of Nephrology, Department of Internal Medicine, Necmettin Erbakan University, Konya, Turkey
\end{abstract}

Received: February 16, 2015 Revised : March 18, 2015 Accepted: June 17, 2015

\section{Correspondence to}

Adem Kucuk, M.D.

Division of Rheumatology, Department of Internal Medicine, Meram School of Medicine, Necmettin Erbakan University, Meram, Konya 42060, Turkey

Tel: +90-332-223-6548

Fax: +90-332-223-6548

E-mail:drademk@yahoo.com

*Current affiliation: Division of Nephrology, Department of Internal Medicine, Sakarya University Research and Training Hospital, Sakarya, Turkey

Background/Aims: Familial Mediterranean fever (FMF) is an autosomal recessive disorder characterized by attacks of fever and diffuse abdominal pain. The primary concern with this presentation is to distinguish it from acute appendicitis promptly. Thus, we aimed to evaluate the role of neutrophil lymphocyte ratio (NLR) to leverage the differential diagnosis of acute FMF attack with histologically proven appendicitis.

Methods: Twenty-three patients with histologically confirmed acute appendicitis and 88 patients with acute attack of FMF were included in the study. NLR, C-reactive protein and other hematologic parameters were compared between the groups.

Results: Neutrophil to lymphocyte ratio was significantly higher in patients with acute appendicitis compared to the FMF attack group ( $8.24 \pm 6.31$ vs. $4.16 \pm 2.44, p$ $=0.007)$. The performance of NLR in diagnosing acute appendicitis with receiver operating characteristic analysis with a cut-off value of 4.03 were; $78 \%$ sensitivity, $62 \%$ specificity, and area under the curve 0.760 ( $95 \%$ confidence interval, 0.655 to $0.8655 ; p<0.001)$.

Conclusions: This study showed that NLR, the simple and readily available inflammatory marker may have a useful role in distinguishing acute FMF attack from acute appendicitis.

Keywords: Neutrophil-to-lymphocyte ratio; Familial Mediterranean fever attack; Acute appendicitis; Abdominal pain; Inflammation

\section{INTRODUCTION}

Familial Mediterranean fever (FMF) is an autosomal recessive disorder characterized by attacks of fever and diffuse abdominal pain. Patients are usually symptom free between the episodes and the frequency of attacks differ from patient to patient [1]. Since FMF usually affects young individuals, a practical challenge emerges when a patient who was not diagnosed with FMF presents classical symptoms of abdominal pain, fever, nausea, and vomiting. The primary concern in this setting is to distinguish FMF attack from appendicitis 
within clinically critical time interval. In case of perforated appendicitis a surgical emergency might become a catastrophic clinical issue due to the delay in diagnosis considering the vice versa situation unless the physician proceeds with a prompt but not prudent decision to operate the patient considering acute appendicitis, will lead to an unnecessary surgery in an otherwise nonsurgical patient facing the risks of operation $[2,3]$. Thus, reliable tools are of crucial importance in determining whether the case presentation is due to FMF attack or acute appendicitis.

Several clinical evidence and biochemical markers have introduced to differentiate the two cases safely and efficiently up to date [1-4]; however, no single laboratory or clinical factor derived concrete differentiative outcomes in this regard. Recent clinical evidence proved the association of neutrophil lymphocyte ratio (NLR) with a number of inflammatory markers. In addition, NLR was shown to be associated with adverse clinical outcomes in various clinical settings [5-10]. Previous studies denoted that FMF attack is associated with an increased value of NLR compared with patients without an active attack [11]. A majority of research revealed that NLR values were significantly different between patients with FMF and healthy subjects showing a positive correlation with NLR subclinical inflammation of attack-free individuals [12]. Previous studies have also emphasized that NLR could be a more sensitive predictor of acute appendicitis compared to C-reactive protein (CRP) and total white blood cell (WBC) count [5,6]. Apart from other causes of lower abdominal quadrant pain, acute FMF attack also presents elevations in acute phase markers including WBC count [7], and solely considering the CRP and WBC count may not be sufficient to differentiate FMF attack and acute appendicitis. To the best of our knowledge, there is no study in the literature analyzing the predictive value of NLR in this regard. Thus, we aimed to evaluate inflammatory markers along with NLR in patients with histologically proven appendicitis and FMF attacks in patients that were already diagnosed with FMF.

\section{METHODS}

This was a retrospective study to compare NLR along with other laboratory parameters between patients who underwent laparotomy for acute appendicitis and FMF attack with pathological evidence. Twenty-three patients with acute appendicitis and 88 patients with acute FMF attack who applied to the emergency services of two hospitals were enrolled in a retrospective manner. All the FMF patients had previously been diagnosed and were administered regular colchicine treatment (none of these attacks were classified as a surgical or another medical condition other than FMF). Patients with atypical attacks and acute abdominal pain due to causes other than FMF attack were excluded from the study. All acute appendicitis cases with suggestive features were practiced laparotomy that had uncomplicated appendicitis morphologically and pathologically. Patients who underwent laparotomy but appeared to have normal appendices were excluded from the study. The patients with diabetes mellitus, coronary heart diseases, thyroidal dysfunction, anemia, B12 and folic acid deficiency, acute/chronic infection, autoimmune disorders, chronic obstructive pulmonary disease, established malignancy, and history of smoking were excluded. Blood counts and CRP values at presentation were obtained from hospitals' electronic database systems. A special effort has been exerted to maintain the blood count and CRP values at the outset of clinical presentation and before surgical intervention in acute appendicitis patients. Institutional Review Board of Necmettin Erbakan University granted approval for the study protocol.

\section{Statistical analysis}

The statistical evaluation was conducted using the SPSS version 16 (SPSS Inc., Chicago, IL, USA). The Kolmogorov-Smirnov test was applied to check the distribution of parameters. Categorical data were presented as numbers and percentages, and continuous data were expressed as mean \pm standard deviation. The difference between the categorical variables was determined using the chisquare test. The independent Student $t$ test was used to determine the difference in normally distributed data, and the Mann-Whitney $U$ test was used for comparison of median for the non-parametrically distributed variables. A $p<0.05$ was considered to be statistically significant. 


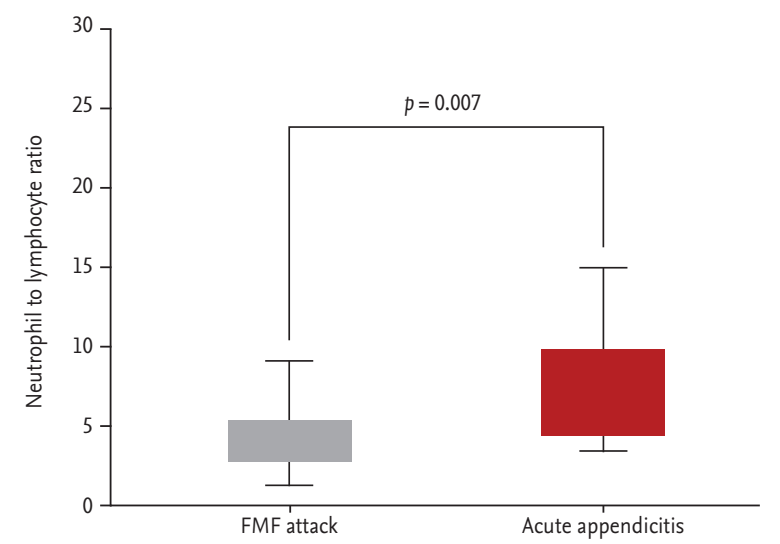

Figure 1. Box and whisker plot showing distribution of neutrophil lymphocyte ratio in patients with acute appendicitis and familial Mediterranean fever (FMF) attack.

\section{RESULTS}

Eighty-eight patients with acute FMF attack ( 55 females, 33 males; mean age, $31.5 \pm 10.4$ years) and 23 patients with acute appendicitis (9 females, 14 males; mean age, 30.8 \pm 11.9 years) were included in the study. The mean age, gender distribution, CRP and hematologic counts of the groups are depicted in Table 1. NLR was significantly higher in patients with acute appendicitis compared with patients presented with an FMF attack $(8.24 \pm 6.31$ vs. $4.16 \pm 2.44, p=0.007$ ) (Fig. 1). On the other hand, there was not a significant difference between the groups in terms of serum CRP values $(39.0 \pm 28.1$ vs. $28.9 \pm 23.3$, $p=0.088$ ). Clinical characteristics and physical examination findings of the patients with FMF attack and acute appendicitis are shown in Table 2. We evaluated the performance of NLR in patients with acute appendicitis with receiver operating characteristic analysis, when cut-off is taken as 4.03, its sensitivity, specificity, and area under the curve were found to be as $78 \%, 62 \%$, and 0.760 , respectively ( $95 \%$ confidence interval, 0.655 to $0.865 ; p<0.001$ ) (Fig. 2).

\section{DISCUSSION}

The prominent finding of this study was that NLR was significantly higher in patients with acute appendicitis compared with patients suffering from an acute FMF attack. To best of our knowledge, this is the first study which utilizes NLR with intend to differ acute appendi-

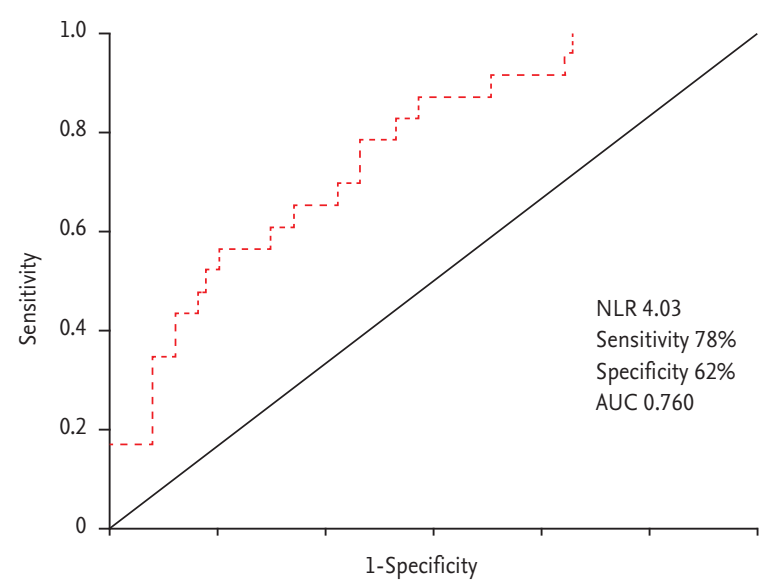

Figure 2. Receiver operating characteristic of neutrophil lymphocyte ratio (NLR) in patients with acute appendicitis. AUC, area under the curve.

citis from an FMF attack.

Presentations of acute appendicitis and FMF attack may have confusing signs and symptoms in some patients especially when the patient with abdominal pain has no previous diagnosis of FMF. Even in patients previously with diagnosed FMF, acute appendicitis may develop and the patient and the caregiver's attribute can direct to a new FMF attack rather than acute appendicitis. In this case, delay in the diagnosis may lead to increased morbidity an even mortality. In areas where FMF is endemic such as Turkey, many FMF patients had undergone unnecessary abdominal exploration for suspected acute appendicitis at some point during the course of their disease [8]. In one study, the frequency of appendectomy found in FMF patients was far above the reported rate in the general population ( $40 \%$ vs. $12 \%$ to $25 \%$ ) [9]. Previous research has also shown that a significant number of patients who presented with acute abdominal pain and other suggestive features for acute appendicitis have been found to have no histologic features of appendicitis when underwent surgery [10]. Therefore, it is a great clinical challenge to accurately and reliably differ acute appendicitis from an FMF attack.

NLR is a novel inflammatory marker and the value of NLR comes from its readily available nature. Complete blood count is almost an inevitable part of modern laboratory evaluation in many clinical settings and NLR can easily be calculated from the blood count. A number of studies showed that NLR was closely correlated with other markers of inflammation. Moreover, in patients 
Table 1. Comparison of the selected laboratory parameters of the study groups

\begin{tabular}{|c|c|c|c|}
\hline Parameter & FMF attack $(\mathrm{n}=88)$ & Acute appendicitis $(n=23)$ & $p$ value \\
\hline Age, yr & $31.5 \pm 10.4$ & $30.8 \pm 11.9$ & 0.806 \\
\hline Sex & & & 0.058 \\
\hline Female & 55 & 9 & \\
\hline Male & 33 & 14 & \\
\hline C-reactive protein, mg/L & $28.9 \pm 23.3$ & $39.0 \pm 28.1$ & 0.088 \\
\hline Erythrocyte sedimentation date, $\mathrm{mm} / \mathrm{hr}$ & $30.4 \pm 20.7$ & $14.1 \pm 11.7$ & $<0.001$ \\
\hline White blood cell count, $\times 10^{3} / \mathrm{L}$ & $9,881 \pm 2,941$ & $13,952 \pm 3,707$ & $<0.001$ \\
\hline Neutrophil count, $/ \mathrm{mm}^{3}$ & $7,097 \pm 2,654$ & $10,873 \pm 3,330$ & $<0.001$ \\
\hline Lymphocyte count, $/ \mathrm{mm}^{3}$ & $1,960 \pm 678$ & $1,712 \pm 730$ & 0.167 \\
\hline Platelet count, $\times 10^{3} / \mu \mathrm{L}$ & $285.1 \pm 67.2$ & $250.3 \pm 45.8$ & 0.027 \\
\hline Neutrophil lymphocyte ratio & $4.16 \pm 2.44$ & $8.24 \pm 6.31$ & 0.007 \\
\hline Hemoglobin, g/dL & $13 \cdot 3 \pm 1.7$ & $13.5 \pm 1.3$ & 0.681 \\
\hline
\end{tabular}

Values are presented as mean \pm SD.

FMF, familial Mediterranean fever.

Table 2. Clinical characteristics and physical examination findings of the patients with FMF attack and acute appendicitis.

\begin{tabular}{lcc}
\hline Characteristic & FMF attack $(\mathrm{n}=88)$ & Acute appendicitis $(\mathrm{n}=23)$ \\
\hline Fever & $86(97.7)$ & $23(100)$ \\
Peritoneal irritation & $85(96.5)$ & $23(100)$ \\
Nausea and vomiting & $19(21.5)$ & $11(47.8)$ \\
Pleuritic pain & $38(43.1)$ & - \\
Pericarditis & $11(12.5)$ & - \\
Arthritis & $5(5.6)$ & - \\
Arthralgia-myalgia & $51(57.9)$ & - \\
Skin lesions & $2(2.2)$ & - \\
\hline
\end{tabular}

Values are presented as number (\%).

FMF, familial Mediterranean fever.

with various cancers [13-15], liver cirrhosis [16], chronic kidney disease [17], and cardiac disease [18,19], NLR could predict adverse clinical outcomes independent of major established risk factors.

Several biochemical parameters have been investigated whether they can reliably differentiate acute appendicitis and acute FMF attack [20]. Serum resistin and visfatin levels have failed to distinguish acute appendicitis from an acute FMF attack [2]. Procalcitonin was found to be significantly higher in patients with appendicitis compared with those of patients with acute FMF attack [4]. However, routine implementation of procalcitonin requires further studies and prohibitively costly yet.
NLR is a simple, non-invasive and cost-effective marker of inflammation in various diseases and is calculated using data obtained from the complete blood count. NLR has been tested to date regarding its ability to accurately diagnose acute appendicitis preoperatively in unselected patients. Almost 20 years ago before NLR became so popular, Goodman et al. [5] declared that $88 \%$ of patients with histologically proven appendicitis had a NLR > 3.5. The authors also concluded that NLR was more sensitive to detect acute appendicitis compared with total WBC count. In a retrospective study in children Yazici et al. [21] found that $90.2 \%$ of the appendicitis group and $12.3 \%$ of the nonspecific abdominal pain group had 
NLR higher than 3.5. Markar et al. [6] evaluated 1,117 cases who underwent appendectomy in which NLR appears to be of greater diagnostic accuracy than either total WBC count or CRP alone. Moreover, some studies reported that NLR could also predict severity of the appendicitis as Ishizuka et al. [22] showed that NLR $>8$ shows a significant association with gangrenous appendicitis in patients undergoing appendectomy. However, none of these studies specifically examined the differential power of NLR between acute appendicitis and FMF attack.

Our results showed that NLR values were significantly higher in patients with acute appendicitis compared with those of acute FMF attack while there was not a significant difference between the groups in terms of serum CRP values. We think that this finding may be particularly important when CRP and clinical findings are not sufficient to safely distinguish acute appendicitis from an FMF attack.

Several limitations of this study are noteworthy. This was a retrospective study and prospective studies recruiting naive patients that admitted emergency services with abdominal pain and/or fever along with other suggestive features of acute appendicitis and/or FMF attack are needed. The differential diagnosis between the two should ideally be based on clinical features at the time of admission and possibly a panel of biochemical tests revealing the degree of inflammation and blood counts. None of the single clinical or laboratory parameters are sensitive or specific enough for concrete diagnosis or exclusion of acute appendicitis or FMF attack. Thus, value of single parameters, in this study example, NLR, should be considered as adjunct to other clinical and laboratory parameters. Prospective studies with large sample size should be designed to achieve a cut-off value or prediction rule based on several clinical and laboratory parameters are definitely needed. Since all FMF patients in our study had their attacks sometime after having diagnosed and received colchicine treatment, these attacks may not be as severe as their first FMF episode which may have caused a more challenging clinical picture. Thus, a prospective study recruiting naive FMF patients would be more ideal to evaluate the role of NLR as a distinction factor. Despite aforementioned limitations, this is the first study showing promising role of NLR at differentiating FMF attack and acute appendicitis.

In conclusion, the main finding of this study can be elaborated as NLR; the simple and cost-effective marker, was significantly higher in patients with acute appendicitis compared with in patients with an FMF attack. We conclude that evaluation of the NLR may have a value in prevention of an unnecessary surgical operation in a non-surgical patient with attendant risks of the operation. Prospective studies are needed to clarify the role of NLR in this situation.

\section{KEY MESSAGE}

1. Acute appendicitis and familial Mediterranean fever (FMF) attack may be confused in emergency department and reliable markers to differentiate the two are required.

2. In this study, neutrophil to lymphocyte ratio was significantly higher in patients with acute appendicitis compared with those of FMF attack.

3. Prospective studies taking clinical presentation as well as several laboratory markers into account would provide additional clues to distinguish the two disorders.

\section{Conflict of interest}

No potential conflict of interest relevant to this article was reported.

\section{Acknowledgments}

Editorial support was given by QA Executive Consultancy Turkey, 2015 (www.QAexecutiveconsultancy.com).

\section{REFERENCES}

1. Onen F. Familial Mediterranean fever. Rheumatol Int 2006;26:489-496.

2. Kisacik B, Erol MF, Yilmaz G, et al. Resistin and visfatin: are they valuable enough to be the differential diagnosis in familial Mediterranean fever with acute appendicitis? Clin Rheumatol 2012;31:225-229.

3. Shohat M, Halpern GJ. Familial Mediterranean fever: a review. Genet Med 2011;13:487-498.

4. Kisacik B, Kalyoncu U, Erol MF, et al. Accurate diagnosis of acute abdomen in FMF and acute appendicitis pa- 
tients: how can we use procalcitonin? Clin Rheumatol 2007;26:2059-2062.

5. Goodman DA, Goodman CB, Monk JS. Use of the neutrophil:lymphocyte ratio in the diagnosis of appendicitis. Am Surg 1995;61:257-259.

6. Markar SR, Karthikesalingam A, Falzon A, Kan Y. The diagnostic value of neutrophil: lymphocyte ratio in adults with suspected acute appendicitis. Acta Chir Belg 2010;110:543-547.

7. Korkmaz C, Ozdogan H, Kasapcopur O, Yazici H. Acute phase response in familial Mediterranean fever. Ann Rheum Dis 2002;61:79-81.

8. Kasifoglu T, Cansu DU, Korkmaz C. Frequency of abdominal surgery in patients with familial Mediterranean fever. Intern Med 2009;48:523-526.

9. Lidar M, Doron A, Kedem R, Yosepovich A, Langevitz P, Livneh A. Appendectomy in familial Mediterranean fever: clinical, genetic and pathological findings. Clin Exp Rheumatol 2008;26:568-573.

10. Chandrasegaram MD, Rothwell LA, An EI, Miller RJ. Pathologies of the appendix: a 10-year review of 4670 appendicectomy specimens. ANZ J Surg 2012;82:844-847.

11. Celikbilek M, Dogan S, Akyol L, et al. Neutrophil-lymphocyte ratio in patients with familial Mediterranean fever. J Clin Lab Anal 2015;29:80-83.

12. Ozer S, Yilmaz R, Sonmezgoz E, et al. Simple markers for subclinical inflammation in patients with familial Mediterranean fever. Med Sci Monit 2015;21:298-303.

13. Lee DY, Hong SW, Chang YG, Lee WY, Lee B. Clinical significance of preoperative inflammatory parameters in gastric cancer patients. J Gastric Cancer 2013;13:111-116.

14. Absenger G, Szkandera J, Pichler M, et al. A derived neu- trophil to lymphocyte ratio predicts clinical outcome in stage II and III colon cancer patients. Br J Cancer 2013;109:395-400.

15. Azuma T, Matayoshi Y, Odani K, et al. Preoperative neutrophil-lymphocyte ratio as an independent prognostic marker for patients with upper urinary tract urothelial carcinoma. Clin Genitourin Cancer 2013;11:337-341.

16. Biyik M, Ucar R, Solak Y, et al. Blood neutrophil-to-lymphocyte ratio independently predicts survival in patients with liver cirrhosis. Eur J Gastroenterol Hepatol 2013;25:435-441.

17. Solak Y, Yilmaz MI, Sonmez A, et al. Neutrophil to lymphocyte ratio independently predicts cardiovascular events in patients with chronic kidney disease. Clin Exp Nephrol 2013;17:532-540.

18. Kaya H, Ertas F, Islamoglu Y, et al. Association between neutrophil to lymphocyte ratio and severity of coronary artery disease. Clin Appl Thromb Hemost 2014;20:50-54.

19. Bhat T, Teli S, Rijal J, et al. Neutrophil to lymphocyte ratio and cardiovascular diseases: a review. Expert Rev Cardiovasc Ther 2013;11:55-59.

20. Bentancur AG, Naveh N, Lancri J, Selah BA, Shtrasburg S, Livneh A. Urine leukotriene $\mathrm{B}_{4}$ in familial Mediterranean fever and other forms of right lower abdominal pain. Acad Emerg Med 2005;12:671-674.

21. Yazici M, Ozkisacik S, Oztan MO, Gursoy H. Neutrophil/ lymphocyte ratio in the diagnosis of childhood appendicitis. Turk J Pediatr 2010;52:400-403.

22. Ishizuka M, Shimizu T, Kubota K. Neutrophil-to-lymphocyte ratio has a close association with gangrenous appendicitis in patients undergoing appendectomy. Int Surg 2012;97:299-304. 